\title{
High-Resolution Secondary Reconstructions with the Use of Flat Panel CT in the Clinical Assessment of Patients with Cochlear Implants
}

\author{
M.S. Pearl, A. Roy, and C.J. Limb
}

\begin{abstract}
SUMMARY: Radiologic assessment of cochlear implants can be limited because of metallic streak artifacts and the high attenuation of the temporal bones. We report on 14 patients with 18 cochlear implants (17 Med-El standard 31.5-mm arrays, 1 Med-El medium 24-mm array) who underwent flat panel CT with the use of high-resolution secondary reconstruction techniques. Flat panel CT depicted the insertion site, cochlear implant course, and all 216 individual electrode contacts. The calculated mean angular insertion depth for standard arrays was $591.9^{\circ}\left(\mathrm{SD}=70.9\right.$; range, $\left.280^{\circ}\right)$. High-resolution secondary reconstructions of the initial flat panel $\mathrm{CT}$ dataset, by use of a manually generated field of view, Hounsfield unit kernel type, and sharp image characteristics, provided high-quality images with improved spatial resolution. Flat panel CT is a promising imaging tool for the postoperative evaluation of cochlear implant placement.
\end{abstract}

ABBREVIATIONS: $\mathrm{Cl}=$ cochlear implant; $\mathrm{EE}=$ edge enhancement; $\mathrm{FPCT}=$ flat panel $\mathrm{CT} ; \mathrm{HU}=$ Hounsfield units; $\mathrm{MSCT}=$ multisection $\mathrm{CT} ; \mathrm{RW}=$ round window

C ochlear implants (CIs) can provide functional restoration of hearing in individuals with profound hearing impairment. Clinical outcomes after implantation vary considerably, however, in the degree and quality of hearing recovery. To assess factors potentially related to $\mathrm{CI}$ outcomes, much attention has been devoted to characterizing the CI electrode location, ${ }^{1,2}$ insertion depth, ${ }^{3}$ scalar localization, ${ }^{4}$ and relationship to the facial nerve canal. ${ }^{2,5}$ These data carry important prognostic information and have implications for surgical approaches, insertion techniques, and programming strategies for optimal auditory nerve stimulation. Although CT is considered the current reference standard method for characterizing CI position, ${ }^{6}$ metallic artifact significantly limits the postoperative imaging evaluation of the electrode array and cochlear anatomy.

Flat panel CT (FPCT) is a relatively new imaging technique that provides CT-like images acquired with a C-arm x-ray system with the use of flat panel image detectors. This technique provides excellent visualization of high-contrast structures with superior

Received August 13, 2013; accepted after revision October 14

From the Division of Interventional Neuroradiology (M.S.P.), and Department of Otolaryngology-Head and Neck Surgery (R.R., C.J.L.), Johns Hopkins University School of Medicine, Baltimore, Maryland; Interventional Neuroradiology (M.S.P.), Children's National Medical Center, Washington, DC; and Peabody Conservatory of Music (C.J.L.), Baltimore, Maryland.

Support for this work was provided by research grants from Med-El Corporation (P.I. Charles Limb) and Siemens Corporate Research (P.I. Monica Pearl).

Please address correspondence to Monica S. Pearl, MD, DABR, Division of Interventional Neuroradiology, The Johns Hopkins Hospital, 1800 Orleans St, Bloomberg Building, 7218, Baltimore, MD 21287; e-mail: msmit135@jhmi.edu

http://dx.doi.org/10.3174/ajnr.A3814 spatial resolution in comparison to multisection CT (MSCT). ${ }^{7}$ Furthermore, secondary reconstruction of the initial FPCT dataset by use of a smaller field of view produces higher-resolution images than can be obtained by standard FPCT reconstructions.

We sought to determine the effectiveness of high-resolution secondary reconstructions of initial FPCT datasets for postoperative CI imaging. We compared 4 different types of image characteristics (very smooth, normal, auto, and sharp) and 2 different kernel types (Hounsfield units [HU] and edge enhancement [EE]) used in the creation of secondary reconstructions to identify the specific reconstruction parameters that lead to optimal CI visualization.

\section{Subjects}

A total of 14 patients with sensorineural hearing loss who underwent previous cochlear implantation with $17 \mathrm{Med}-\mathrm{El}$ standard 12-electrode contact arrays (31.5-mm linear insertion length, 2.4 $\mathrm{mm}$ between contacts) and $1 \mathrm{Med}-\mathrm{El}$ medium 12-electrode contact array (24-mm linear insertion length, $1.9 \mathrm{~mm}$ between contacts) (Med-El, Innsbruck, Austria) were included (Table 1). Subjects included 8 men and 6 women with a mean age of 53 years (range, 21-62 years). A standard posterior tympanotomy approach was used in all cases. The implantation approaches were mixed and included pure round window (RW) insertions as well as cochleostomies just anterior to the RW with or without extension into the RW.

All patients gave written informed consent to participate in this institutional review board-approved study. 
Table 1: Demographic information for patients with cochlear implants

\begin{tabular}{|c|c|c|c|c|c|c|c|}
\hline Subject & Sex & Age, $y$ & Etiology & Implant Device & Implant Side & Fig 5 & RW Insertion \\
\hline 1 & $\mathrm{~F}$ & 59 & Meniere & Med-El Sonata, Standard Array & Right & B & \\
\hline 2 & $\mathrm{~F}$ & 48 & Autoimmune & Med-El Sonata, Standard Array & Right & $\mathrm{H}$ & RW \\
\hline 3 & M & 62 & Meniere & Med-El Sonata, Standard Array & Left & $\mathrm{R}$ & RW \\
\hline \multirow[t]{2}{*}{$4^{a}$} & M & 51 & Idiopathic & Med-El Concert, Standard Array & Right & $\mathrm{F}$ & RW \\
\hline & & & & Med-El Concert, Standard Array & Left & L & \\
\hline 5 & $\mathrm{~F}$ & 54 & Hereditary & Med-El Concert, Standard Array & Left & $Q$ & \\
\hline 6 & $\mathrm{~F}$ & 21 & Idiopathic & Med-El Sonata, Standard Array & Left & $P$ & \\
\hline \multirow[t]{2}{*}{$7^{\mathrm{a}}$} & $M$ & 59 & Meniere & Med-El Concert, Standard Array & Right & $\mathrm{D}$ & \\
\hline & & & & Med-El Sonata, Standard Array & Left & M & \\
\hline \multirow[t]{2}{*}{$8^{a}$} & M & 62 & Idiopathic & Med-El Sonata, Standard Array & Right & $C$ & RW \\
\hline & & & & Med-El Sonata, Standard Array & Left & $J$ & \\
\hline 9 & $\mathrm{~F}$ & 51 & Idiopathic & Med-El Concert, Standard Array & Right & G & RW \\
\hline 10 & M & 61 & Meniere & Med-El Concert, Standard Array & Left & $\mathrm{O}$ & \\
\hline \multirow[t]{2}{*}{$11^{\mathrm{a}}$} & M & 57 & Idiopathic & Med-El Sonata, Standard Array & Right & $\mathrm{E}$ & \\
\hline & & & & Med-El Sonata, Standard Array & Left & K & \\
\hline 12 & M & 49 & Meningitis & Med-El Concert, Standard Array & Right & $A$ & \\
\hline 13 & M & 61 & Viral & Med-El Sonata, Standard Array & Left & $\mathrm{N}$ & \\
\hline 14 & $\mathrm{~F}$ & 50 & Idiopathic & Med-El Concert, Medium Array & Right & I & \\
\hline
\end{tabular}

Note:-Demographic information for all 14 patients with cochlear implants include sex, age, etiology of hearing loss, type of Cl, laterality, and corresponding image in Fig 5 . Round window insertions are designated by RW.

a Subjects with bilateral Cls.
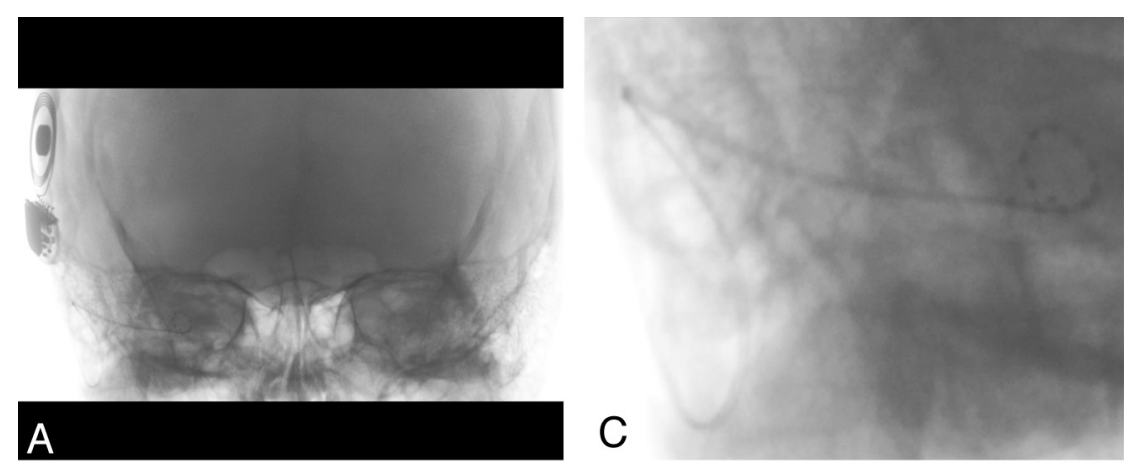

software; Siemens). High-resolution secondary reconstructions (Fig $1 C-D$ ) were created with the use of the following parameters: manually generated voxels of interest to include only the electrode array; voxel size, $0.07-0.08 \mathrm{~mm}, 512 \times 512$ section matrix; $\mathrm{HU}$ and $\mathrm{EE}$ kernel types; and very smooth, normal, auto, and sharp image characteristics.

An interventional neuroradiologist with fellowship training and subspecialty certification in diagnostic neuroradiology (M.S.P.) performed the postprocessing. All images were evaluated by all 3 authors: an interventional neuroradiologist with subspecialty certification in diagnostic neuroradiology (M.S.P.); a neuroscientist with $>4$ years of research dedicated to CIs (A.R.); and a neurotologist who is a $\mathrm{CI}$ specialist and has $>15$ years of experience in the field of CI (C.J.L.).

\section{Reconstruction Parameters}

FIG 1. Flat panel CT of a right-sided cochlear implant. $A$, Collimated fluoroscopic acquisition for a 20-second FPCT of a right-sided $\mathrm{CI}$. The skull above and below is excluded from the initial acquisition. B, Example of a coronal oblique image acquired after the default reconstruction. $C$, Secondary reconstruction by use of a manually generated voxel of interest is created to include only the electrode array. D, Higher-resolution coronal oblique image is generated after the secondary reconstruction.

\section{FPCT Imaging Protocol}

All subjects underwent FPCT (DynaCT; Siemens, Erlangen, Germany) evaluation on a flat-panel angiography system (Axiom Artis Zee, Siemens) with the use of commercially available software (Syngo DynaCT, Siemens). A collimated 20-second FPCT of the head (Fig $1 A$ ) was performed by use of the following parameters: $109 \mathrm{kV}$, small focus, $200^{\circ}$ degree rotation angle, and $0.4^{\circ}$ degree/ frame angulation step. Postprocessing was performed on a commercially available workstation (Leonardo DynaCT, InSpace 3D
The secondary reconstruction parameters available for postprocessing included kernel type (HU and EE) and image characteristic (very smooth, normal, auto, and sharp). Each kernel type was paired with each type of image characteristic to create the secondary reconstructions by use of a smaller manually generated VOI.

For each reconstruction, 2D multiplanar images were created. Coronal oblique images in the plane of the array were generated by aligning the multiplanar reconstruction axes on the axial and sagittal planes parallel to the basal turn and perpendicular to the modiolus (Fig 2). The section thickness was set to $3 \mathrm{~mm}$ to include all electrode contacts on one image. The 

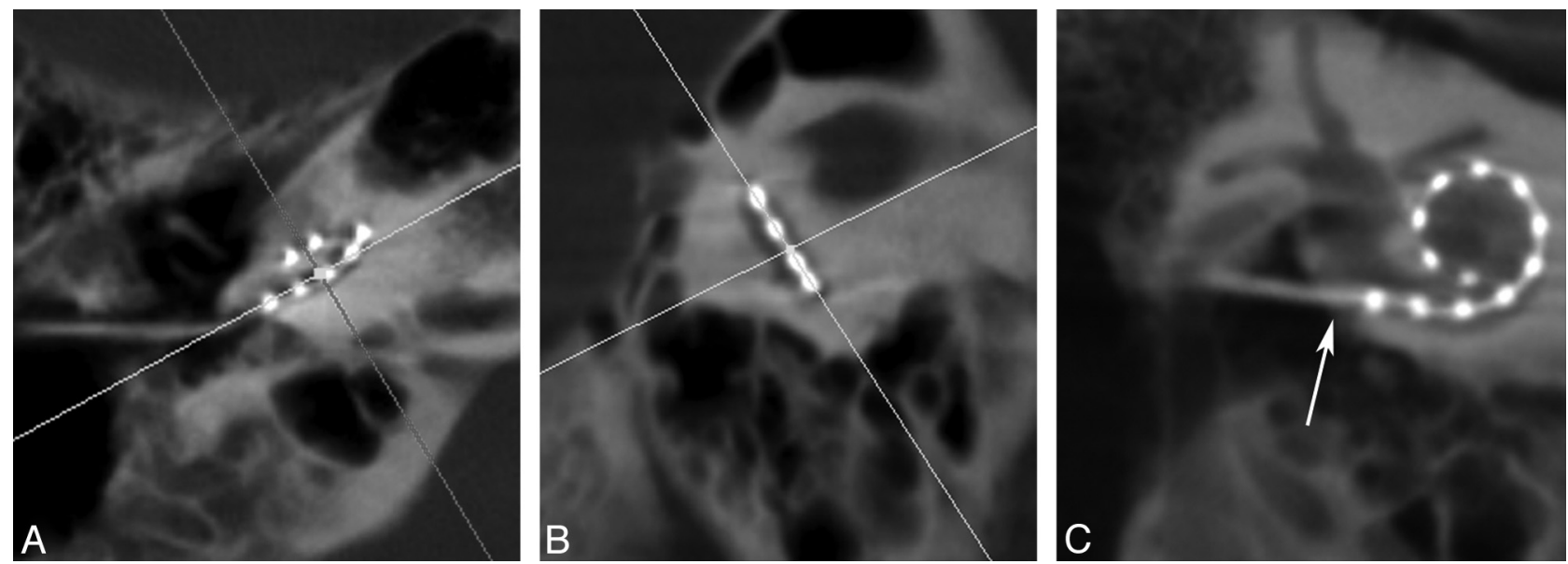

FIG 2. Multiplanar reconstruction images of a right-sided cochlear implant. Multiplanar reconstruction axes are aligned parallel to the basal turn on the axial (A) and sagittal (B) planes to generate a coronal oblique (C) image of the $\mathrm{Cl}$. This image is rotated slightly to visualize the vestibule and superior semicircular canal. Arrow in (C) denotes the insertion point.
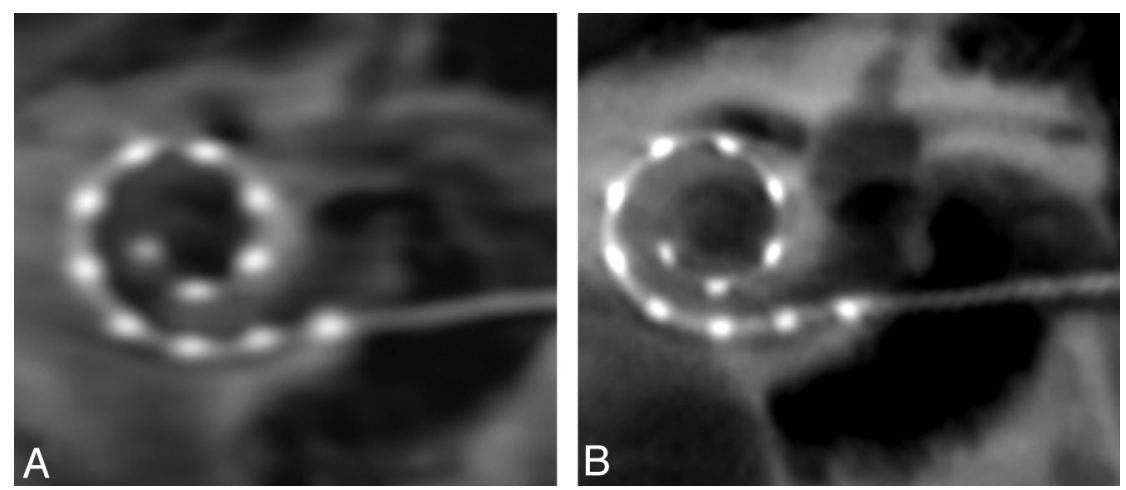

FIG 3. Secondary reconstructions comparing edge enhancement (A) and Hounsfield unit (HU) (B) kernel type show more distinct individual electrode contacts and osseous structures on the image generated by HU kernel type.

window width and contrast level were adjusted until both the individual electrode contacts and surrounding labyrinthine structures were visualized.

The individual electrode contacts appeared less distinct by use of the EE kernel type in comparison to the HU kernel type (Fig 3). All individual electrode contacts could be identified by means of all 4 types of image characteristics; however, the very smooth and normal types created images whose electrodes and surrounding osseous structures were blurry. More distinct contacts and osseous structures were apparent with the auto and sharp image characteristics. The combination of reconstruction parameters that produced the clearest depiction of the electrode array and surrounding labyrinthine structures were HU kernel type and sharp image characteristics (Fig 4).

\section{Visualization of Insertion Point and Individual Electrode Contacts}

FPCT depicted all 18 insertion points and distinguished all 216 individual electrode contacts for all 14 patients (Fig $5 A-R$ ). This includes 1 patient (Fig 5I) with a Med-El medium 12-electrode contact array. A standard posterior tympanotomy approach was used in all subjects. Five of the 18 CIs were pure RW insertions (Table 1).

\section{Measuring Angular Insertion Depth}

The 2D MPR section thickness was reduced to $0.1 \mathrm{~mm}$ to determine the electrode array insertion point, defined by the entrance of the electrode array from the middle ear into the lumen of the scala tympani. This was determined in the axial images at the air-to-bone interface along the outer margin of the electrode and then confirmed in the sagittal and coronal planes. The insertion point was designated in the coronal images and used as the first reference point for measuring angular insertion depth. With the use of a 3 -mm section thickness, the 3 most apical electrodes were identified and used to create a circle depicting their course. A line drawn from the insertion point to the center of this circle served as the reference (zero degree) line. The angle $\theta$ between the most apical electrode and the reference line was calculated (Fig 6). This angle was subtracted from or added to 360 or 720 , depending on the relationship between the apical electrode and the number of turns across the reference line (Table 2).

The mean apical electrode insertion depth for the standard $31.5-\mathrm{mm}$ array was $519.1^{\circ}\left(\mathrm{SD}=70.8\right.$; range, $\left.468.8-749.0^{\circ}\right)$. The apical insertion depth for the medium $24-\mathrm{mm}$ array was $424.0^{\circ}$ (Fig 7). There was no significant difference in the angular insertion depth between male (mean $=593.3 ; \mathrm{SD}=67.3)$ and female patients $($ mean $=541.9 ; \mathrm{SD}=47.9), t(11)=1.81, P=.10$, 2-tailed; however, this observation may be limited because of the small sample size in each group. Given the idiopathic etiology for hearing loss in $43 \%$ of our subjects ( 6 of 14; not an uncommon finding in this patient population) we did not specifically compare etiology with implant depth.

\section{DISCUSSION}

Advances in CI design and implantation have been limited by the inability to assess individual electrode contact placement, reliably calculate angular insertion depth, and accurately determine the 

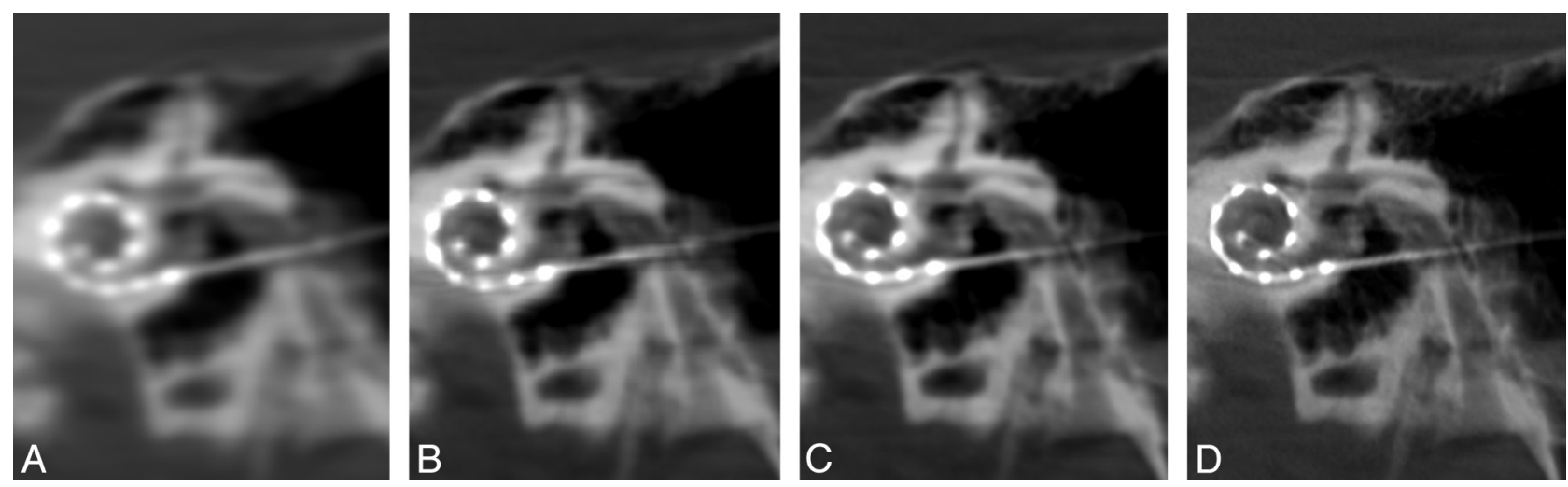

FIG 4. Coronal oblique secondary reconstructions of the same patient with cochlear implants. These images illustrate the variable image quality obtained with identical 3-mm section thickness, window width (3681), contrast (1246), and kernel type (HU). Note the blurring and poor visualization of the electrode contacts with the very smooth $(A)$ setting. A sharp $(D)$ parameter produces optimal images with well-defined osseous structures and individual electrode contacts. (A) Very smooth, (B) normal, (C) auto, (D) sharp.

relationship of the electrode array to adjacent labyrinthine structures. These data provide important prognostic information and may play a critical role in CI outcomes. ${ }^{8}$

Imaging evaluation of postoperative CI placement was initially performed with plain radiographs in the Stenvers projection. ${ }^{9,10}$ MSCT replaced this method and has since become the reference standard imaging technique for assessing the electrode array location relative to intracochlear scalae, the electrode-modiolar interval, and the proximity of the electrode to the fallopian canal. ${ }^{6} \mathrm{CT}$ offers the ability to provide $3 \mathrm{D}$ positional information and excellent contrast for different tissue types. ${ }^{11}$ Despite these advantages, however, attenuated bone structures within the acquisition field ${ }^{12}$ and metallic artifacts severely limit the visibility of electrode contacts and adjacent structures. ${ }^{10,13,14}$

FPCT is a relatively new imaging technique that creates less streak artifacts and offers higher spatial resolution than MSCT (cubic voxel size of approximately $0.07 \mathrm{~mm}$ versus $0.4-0.6 \mathrm{~mm}$, respectively). ${ }^{7}$ These CT-like images can discern individual electrode contacts, often not possible on MSCT. ${ }^{2,7}$ In addition, FPCT has demonstrated improved imaging of the cochlea and facial nerve canal, enabling identification of electrode contacts in close proximity to the fallopian canal and therefore most prone to induce inadvertent stimulation of the facial nerve. ${ }^{2,15}$

Initial studies with the use of FPCT in the evaluation of CIs were performed with the use of cadaveric-implanted temporal bones. ${ }^{2,6,7}$ The authors noted potential limitations caused by the use of temporal bone specimens rather than whole skulls, which were thought to be more challenging for imaging purposes. ${ }^{2}$ Only recently have patients with CIs been evaluated by FPCT. ${ }^{3,16}$ Our study represents one of the larger series to date of CI assessment with FPCT (14 patients, 18 electrode arrays), and the only one to explore optimal reconstruction parameters, including secondary reconstruction techniques. In our series, FPCT was able to identify all 18 insertion points and all 216 individual electrode contacts as well as to identify surrounding labyrinthine structures that were not degraded by streak artifact. Secondary reconstructions of the initial FPCT dataset by use of a VOI to include only the electrode array created voxel sizes of $0.07-0.08 \mathrm{~mm}$, allowing for higher-resolution images than initial reconstructions (Fig $1 B-D$ ). These datasets were optimized by use of an HU kernel type and sharp image characteristic, which enabled the distinct identification of individual electrode contacts and surrounding osseous and soft tissue structures. Figure 4 highlights the range of image quality obtained with different postprocessing algorithms and shows the advantages of the "sharp" algorithm, with better delineation of osseous structures and less blurring of the image.

FPCT could not clearly detect the basilar membrane to designate exact scalar location, which is consistent with previous reports. ${ }^{2}$ CT methods for this designation use oblique coronal images reconstructed perpendicular to the basal turn and parallel to the modiolus. The scalar position, however, is inferred in relation to the cochlear walls ${ }^{17}$ because the image resolution is insufficient to visualize the osseous spiral lamina and basilar membrane. ${ }^{18}$ Although we could infer the scalar position on the basis of the relative location of the $\mathrm{CI}$ in the cochlear chamber, this was not specifically attempted because definitive location could not be made or compared with any other standard. Additionally, given that an electrode may penetrate the basilar membrane and traverse $>1$ scalae, it is difficult to assume true location without visualizing distinct landmarks. Scalar localization will be the subject of future research as reconstruction algorithms and other advanced postprocessing techniques continue to improve. Whereas some reports cite the ability to clearly detect scalar position of the $\mathrm{CI}$, the assessment of positioning within the scala tympani was primarily demonstrated in ex vivo specimens. ${ }^{16}$

The limitations of our report include the evaluation of only 1 CI manufacturer (Med-El; chosen for consistency during development of the imaging protocol), and the predominant assessment of CIs with electrode contacts spaced $2.4 \mathrm{~mm}$ apart (Med-El standard array), which are farther apart than some arrays currently available through other manufacturers. The distinct identification of individual electrode contacts was easily achievable, suggesting that this method should also distinguish more closely spaced contacts. Patient 14 (Fig 5I), implanted with the Med-El medium 12-electrode contact array, demonstrates the feasibility of visualizing electrode contacts spaced $1.9 \mathrm{~mm}$ apart. Currently planned studies will apply the FPCT technology with high-resolution secondary reconstruction algorithms to investigate other electrode designs and CI manufacturers. 

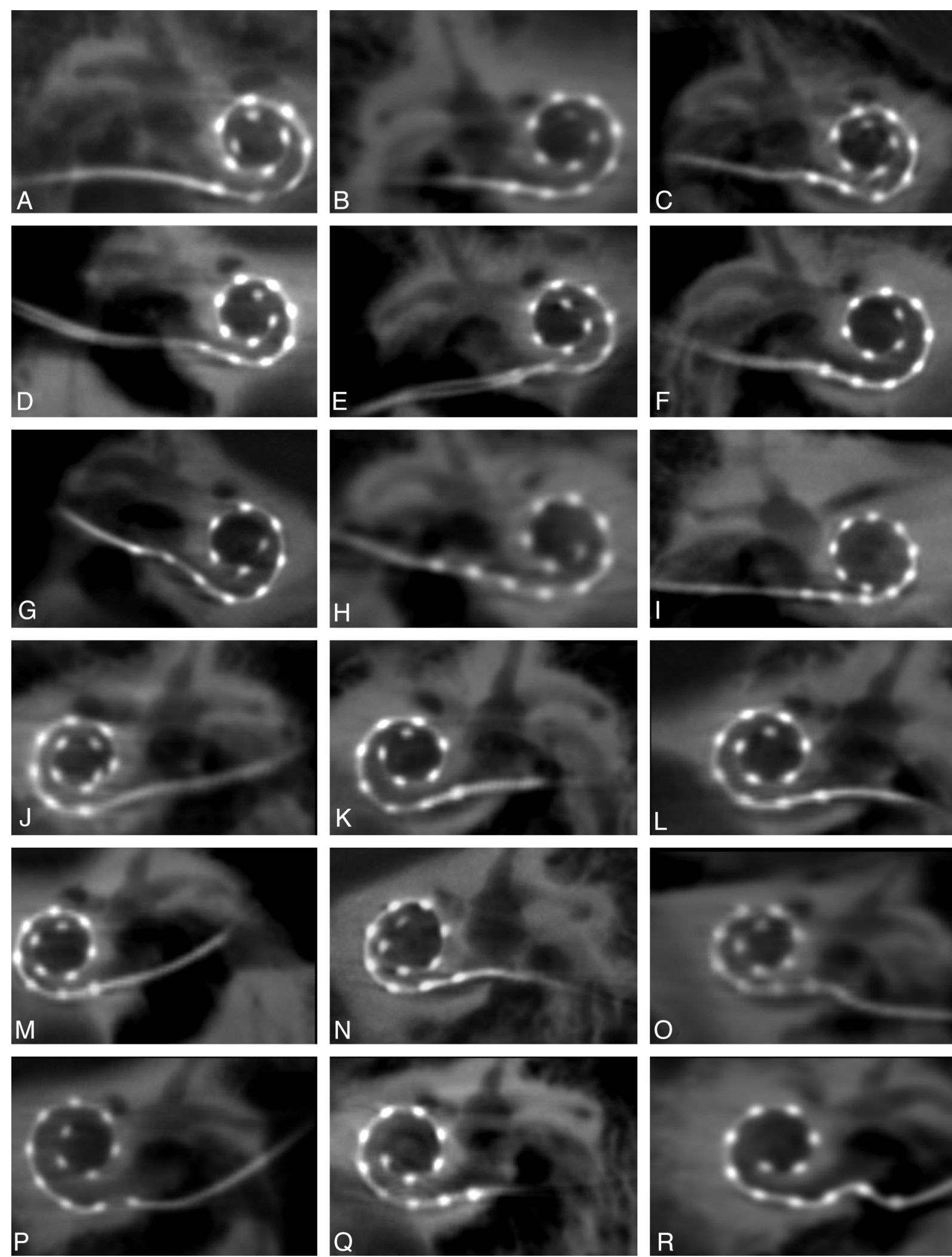

FIG 5. High-resolution secondary reconstructions of all 14 patients and 18 cochlear implants (Cls). $A-I$, Nine right-sided Cls, including 1 patient (I) with an implanted medium array, are shown in the coronal oblique plane. $J-R$, Nine left-sided Cls are depicted, all of which are the standard length array. Images are arranged in order of descending angular insertion depth. All 216 individual electrode contacts are clearly visualized in all patients. 


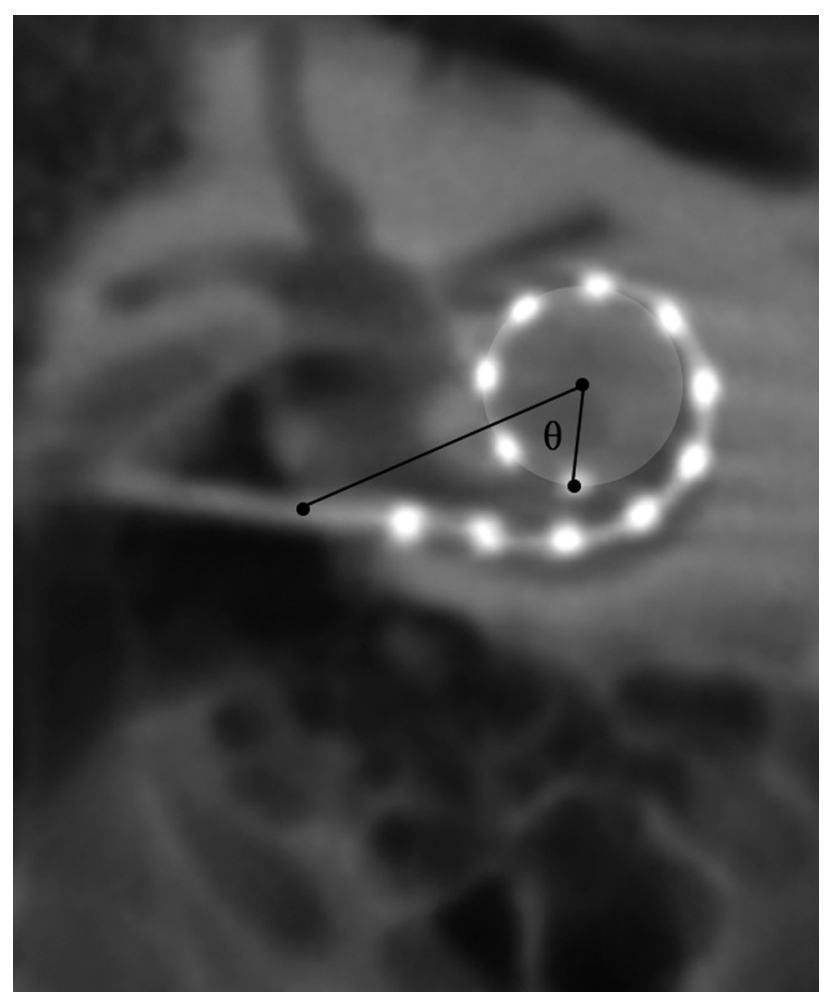

FIG 6. Method for calculating the angular insertion depth of the most apical electrode. The insertion point, as determined by the axial and sagittal images (not shown) is designated in the coronal oblique image. A circle whose outer circumference represents the trajectory of the 3 most apical electrodes is drawn. A reference line is drawn between the insertion point and the center of this circle. The most apical electrode is then identified and the angle $\theta$ is calculated between the electrode and the reference line. In this example, angular insertion depth is calculated by $360 \pm \theta$.

Table 2: Method for calculating angular insertion depth

\begin{tabular}{cc}
\hline No. of Complete Turns & Angular Insertion Depth \\
\hline$<1$ & $360-\theta$ \\
$>1,<1.5$ & $360+\theta$ \\
$>1.5,<2$ & $720-\theta$ \\
$>2$ & $720+\theta$ \\
\hline
\end{tabular}

Note:-Angular insertion depth calculations: The angular insertion depth for apical electrodes coursing $<1$ complete turn in relation to the zero degree line is calculated by $360-\theta$; for $>1$ turn but $<540^{\circ}$ (1.5 turns), insertion depth equals $360+\theta$; for $>1.5$ turns but $<2$ turns, insertion depth equals $720-\theta$. For $>2$ turns, insertion depth equals $720+\theta$

\section{CONCLUSIONS}

FPCT evaluations of postoperative CIs are easy to perform, produce high-resolution images, and are able to depict all individual electrode contacts, thus enabling measurement of the angular insertion depth of the most apical electrode contacts. Optimal imaging methods use a high-resolution secondary reconstruction algorithm with a manually generated small VOI (voxel size, 0.07$0.08 \mathrm{~mm}$ ), HU kernel type, and sharp image characteristic. These advances in CI imaging may lead to a better understanding of the relationship between cochlear anatomy, CI electrode placement, and auditory performance.

Disclosures: Monica Pearl—RELATED: Grant: Siemens Corporate Research, ${ }^{\star}$ Comments: Research grant, PI Monica Pearl; UNRELATED: Siemens Corporate Research,* Comments: Previous research support; Travel/Accommodations/Meeting Expenses Unrelated to Activities Listed: Siemens Corporate Research, ${ }^{\star}$ Comments:

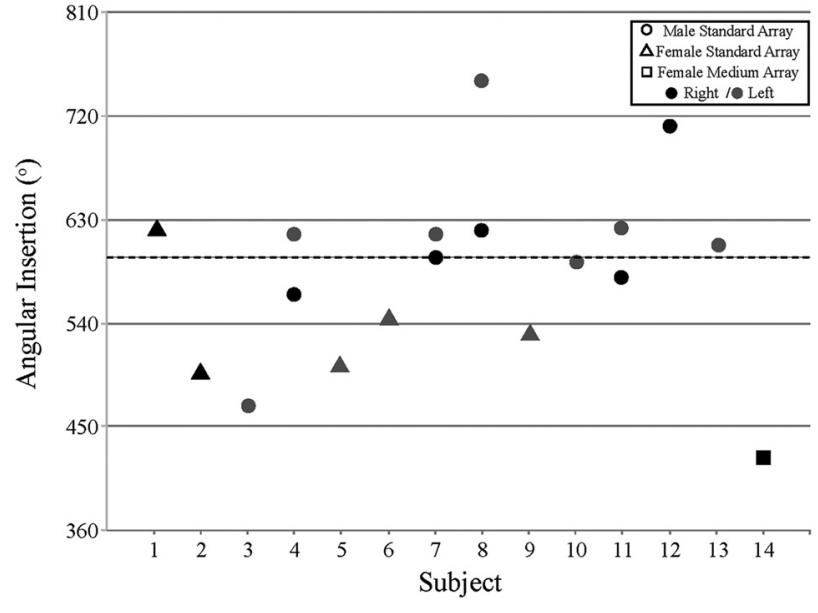

FIG 7. Measured angular insertion depth of the most apical electrodes. Circles and triangles depict angular insertion depth for male and female patients with Med-El 31.5-mm standard array, respectively. Square refers to the single Med-El 24-mm medium array patient. Black filled shapes depict a right implanted ear; gray filled shapes depict a left implanted ear.

Travel expenses for ASNR meetings to present research. Alexis Roy-RELATED: Grant: Med-El Corporation, Comments: Med-El Corporation provided a grant to Johns Hopkins University for part of this work; however, they were not involved in data collection, analyses, or manuscript preparation. Charles Limb-RELATED Grant: Med-El Corporation, Comments: Grant to study musical sound quality in cochlear implant users; UNRELATED: Consultancy: Advanced Bionics Corporation,* Comments: Research support given for project studying music perception in cochlear implant users; Expert Testimony: Karp Frosh law offices, Comments: Money received for expert case review; testimony pending; Payment for Lectures (including service on speakers bureaus): Academic and private honoraria for lectures on music cognition. Comments: No speakers bureaus; Royalties: Up-To-Date section editor, Chronic Otitis Media (*money paid to institution).

\section{REFERENCES}

1. Teymouri J, Hullar TE, Holden TA, et al. Verification of computed tomographic estimates of cochlear implant array position: a micro-CT and histologic analysis. Otol Neurotol 2011;32:980-06

2. Bartling SH, Gupta R, Torkos A, et al. Flat-panel volume computed tomography for cochlear implant electrode array examination in isolated temporal bone specimens. Otol Neurotol 2006;27:491-98

3. Trieger A, Schulze A, Schneider M, et al. In vivo measurements of the insertion depth of cochlear implant arrays using flat-panel volume computed tomography. Otol Neurotol 2011;32:152-57

4. Lane JI, Driscoll CL, Witte RJ, et al. Scalar localization of the electrode array after cochlear implantation: a cadaveric validation study comparing 64-slice multidetector computed tomography with microcomputed tomography. Otol Neurotol 2007;28:191-94

5. Vanpoucke F, Zarowski A, Casselman J, et al. The facial nerve canal: an important cochlear conduction path revealed by Clarion electrical field imaging. Otol Neurotol 2004;25:282-89

6. Zeitler DM, Wang KH, Prasad RS, et al. Flat-panel computed tomography versus multislice computed tomography to evaluate cochlear implant positioning. Cochlear Implants Int 2011;12:216-22

7. Struffert T, Hertel V, Kyriakou Y, et al. Imaging of cochlear implant electrode array with flat-detector CT and conventional multislice CT: comparison of image quality and radiation dose. Acta Otolaryngol 2010;130:443-52

8. Finley CC, Holden TA, Holden LK, et al. Role of electrode placement as a contributor to variability in cochlear implant outcomes. Otol Neurotol 2008;29:920-28 
9. Cohen LT, Xu J, Xu SA, et al. Improved and simplified methods for specifying positions of the electrode bands of a cochlear implant array. Am J Otol 1996;17:859-65

10. Xu J, Xu SA, Cohen LT, et al. Cochlear view: post-operative radiography for cochlear implantation. Am J Otol 2000;21:49-56

11. Skinner MW, Ketten DR, Vannier MW, et al. Determination of the position of nucleus cochlear implant electrodes in the inner ear. Am J Otol 1994;15:644-51

12. Whiting BR, Holden TA, Brunsden BS, et al. Use of computed tomography scans for cochlear implants. J Digit Imaging 2008;21: $323-28$

13. Whiting BR, Bae KT, Skinner MW. Cochlear implants: three-dimensional localization by means of coregistration of $\mathrm{CT}$ and conventional radiographs. Radiology 2001;221:543-49
14. van Wermeskerken GK, van Olphen AF, Graamans K. Imaging of electrode position in relation to electrode functioning after cochlear implantation. Eur Arch Otorhinolaryngol 2009;266:1527-31

15. Bigelow DC, Kay DJ, Rafter KO, et al. Facial nerve stimulation from cochlear implants. Am J Otol 1998;19:163-69

16. Arweiler-Harbeck D, Monninghoff C, Greve J, et al. Imaging of electrode position after cochlear implantation with flat panel CT. ISRN Otolaryngol 2012;2012:728205

17. Ruivo J, Mermuys K, Bacher K, et al. Cone beam computed tomography, a low-dose imaging technique in the post-operative assessment of cochlear implantation. Otol Neurotol 2009;30:299-303

18. Verbist BM, Frijns JH, Geleijns J, et al. Multisection CT as a valuable tool in the post-operative assessment of cochlear implant patients. AJNR Am J Neuroradiol 2005;26:424-29 